\title{
Industry helps mobilize DoD biological expertise
}

Recent bioterrorist events have highlighted the vulnerability of the United States when it comes to biological warfare, indicating the need for advanced diagnostics and better civilian responses. Poor technology transfer between the US Department of Defense (DoD; Arlington, VA) and civilian government agencies, as well as the reluctance of industry to embark on collaborations in areas with limited markets, have hindered progress.

When two bioterrorist suspects were arrested near Las Vegas in February, Federal Bureau of Investigation (FBI; Washington, DC) agents called on experts from the DoD and civilian health agencies to help analyze the suspected deadly anthrax materials. It was several days before DoD experts several thousand miles away at the US Army Research Institute of Infectious Diseases (USAMRIID, Frederick, MD) could use their impressive biological agent detection technology systems to confirm that the materials were actually harmless vaccine samples.

Cumbersome crossover between US government departments is problematic and was confirmed in a report issued earlier this year by the Institute of Medicine and the National Research Council (Washington, DC), "Improving Civilian Medical Response to Chemical or Biological Terrorist Incidents," which concludes that measures for detecting and counteracting biological warfare activities are not well developed.

The report recommended that the federal government should go beyond supporting biological warfare defense-related research. It should provide increased support for the health surveillance infrastructure, surveying the capacity of major hospitals to deal with biological terrorist incidents, and working with the Centers for Disease Control and Prevention (CDC; Atlanta, GA) and state health officials to systematize information for dealing with suspected biological agents. Furthermore, the report says, federal government should work with the US Food and Drug Administration (FDA; Rockville, MD) on guidelines for using unapproved investigational products to counteract an incident. All of that would require substantial new resources.

A broad directive issued by the Clinton administration in June 1996 asks the DoD to conduct global surveillance of emerging infectious diseases as a matter of national security. This is seen by some officials at the $\mathrm{DoD}$ as a worrying "profound expansion of our mission." Increasing pressure on DoD programs to provide expertise and materials for broader civilian use is further taxing pro- grams that are already inadequately funded and have become understaffed, they say.

Although several civilian agencies such as the FBI and the CDC have primary responsibility for dealing with biological warfare incidents, specialists from USAMRIID and DoD departments are called on when terrorists brandish potentially dangerous biological agents within the US domestic scene. However, joint efforts to develop effective response plans are thwarted by inadequate budgets, divergent missions, and bureaucracy, according to officials.

Even access to specialized high-tech equipment developed by the DoD has been limited for civilian agencies. For example, a miniaturized PCR-based device for detecting biological warfare agents in the field has been developed by a team at USAMRIID in collaboration with the Armed Forces Pathology Institute (Washington, DC) and the Lawrence Berkeley National Laboratory (Berkeley, CA). Although DoD researchers have demonstrated a prototype to their CDC colleagues, it is currently not available to the $\mathrm{CDC}$, leaving officials there both frustrated and envious.

Things are starting to change, however. Under the Federal Technology Transfer Act, the biological miniaturization specialist company, Cepheid (Sunnyvale, CA) has received contract support from the DoD, including from the Defense Advanced Research Projects Agency (Arlington, VA; see Nature Biotechnology 15:611-612, 1997), and is expected to begin marketing a fairly simple version of this device for research applications sometime next year and soon thereafter for clinical uses, says Erik Henchal, chief of the USAMRIID Diagnostic Systems Division.

Technology transfer inertia is not the only difficulty facing DoD-originated research and development. DoD has, for instance, faced obstacles within its vaccine development programs. Moving vaccines against biological warfare agents through clinical trials is not straightforward because the usual procedures cannot be followed. Conducting placebo-controlled clinical trials in order to prove safety and efficacy, for example, is impossible because almost no one normally contracts biological warfare diseases.

According to former USAMRIID commander David Franz, who is now deputy commander of the US Army Medical Research and Material Command, USAMRIID researchers have developed more than a dozen recombinant-DNA vaccines during the past decade to protect US troops against potential biological warfare pathogens, tox- ins, or exotic infectious diseases. Not only has regulatory evaluation proved difficult, it has not been easy to strike partnerships with industry to develop such products whose markets are considered, under ordinary circumstances, to be very limited. As a result, the DoD has been criticized for using (or planning to use) some unlicensed/experimental vaccines on military troops.

These frustrations have, in part, prompted a $\$ 322$ million, 10-year contract program between the DoD and DynPort, a subsidiary of DynCorp (Reston, VA) for moving experimental vaccines through approval. DynPort will oversee a consortium of companiesincluding Southern Research Institute (Frederick, MD), McKesson Bioservices (Rockville, MD), and several potential manufacturers such as Greer Laboratories (Lenoir, $\mathrm{NC}$ ) - and will receive experimental medical products from research groups. DynPort intends to work with the US Army Medical Research and Development Command to establish safety and efficacy of such products for FDA approval, and will then produce, test, and distribute the products.

Jeffrey L. Fox

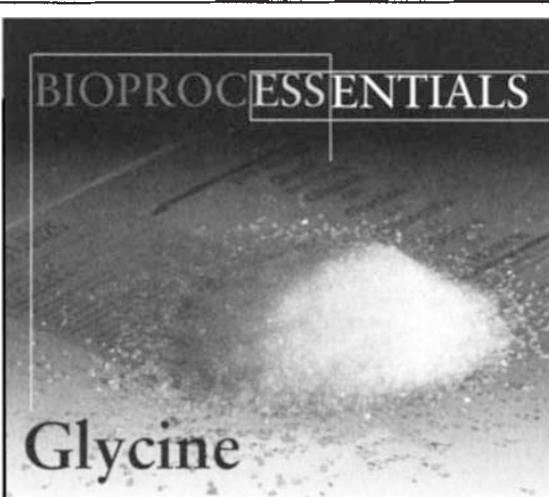

is frequently used in bioprocessing, e.g. as: - A buffer

- A component of fermentation and cell culture media

- For the stabilisation of protein preparations.

When using glycine and other amino acids from Merck KGaA, you will always be on the safe side; only selected raw materials, essentially of non-animal origin, are used in the fermentation and synthetic production processes involved. The amino acids produced in this way not only fulfil the requirements of all important pharmacopoeias (USP, DAB, Ph Eur, BP) but in fact exceed them in many of the specifications.

Merck KGaA Darmstadt · Germany 